\title{
Effect of surface stress and irregularity of the interface on the propagation of SH-waves in the magneto-elastic crustal layer based on a solid semi space
}

\author{
D P ACHARYA* and INDRAJIT ROY** \\ *109/3, Kailash Roy Chowdhury Road, Barrackpore, Kolkata 700120 \\ **B Brahmapara, Simurali, Nadia 741248 \\ e-mail: acharyadp_05@rediffmail.com; mecroy@yahoo.com
}

MS received 27 May 2007; revised 4 March 2009

\begin{abstract}
The object of the present paper is to investigate plane SH waves through a magneto-elastic crustal layer based over an elastic, solid semi space under the influence of surface stress on the free surface of the crustal layer and irregularity of the interface. Two types of irregularities of the interface namely, rectangular and parabolic have been considered. Modulations of wave velocity due to the presence of surface stress, irregularity and the magnetic field have been studied separately. Their combined effect has also been investigated. Graphs are drawn to highlight some important peculiarities. It is observed that surface stress, irregularity and magnetic field have their respective role to play in the propagation of $\mathrm{SH}$ waves in the crustal layer. Further modulation of wave velocity occurs due to their combined effect.
\end{abstract}

Keywords. Surface stress; irregularity of the interface; magneto-elastic crustal layer; dispersion equation, $\mathrm{SH}$-waves, wave velocity equation.

\section{Introduction}

From the literatures presented by Gurtin \& Murdoch (1976), Chandrasekharaiah (1987a), and some other authors (Plaster 1972; Pal et al 1997; Gurtin 1972) it is known that surface stress plays a vital role in the propagation of waves due to the fact that the surface of a body exhibits properties quite different from those associated with the interior of the medium. For example, surface tension which is generally present in liquid may be considered as a particular case of surface stress. Presence of surface stress on the boundary of any body has been detected in some particular type of crystals where the order of magnitude agrees with the predictions made by the molecular theory (Gurtin \& Murdoch 1976). Compressive surface stress is involved in the case of short peening of ductile metals (Gurtin \& Murdoch 1976). Visible strain arises due to this process. This process is used in the shaping of aircraft wing panels

${ }^{*}$ For correspondence 
(Gurtin \& Murdoch 1976). A few problems of the propagation of plane waves in homogeneous and isotropic materials were considered by Gurtin \& Murdoch (1976). Though the concept of surface stress is comparatively of recent origin, a few authors (Chandrasekharaiah 1987a, Plaster 1972) investigated problems which are based on the effect of surface stress from different angles. Pal et al (1997) investigated the effect of surface stress on the propagation of surface waves.

Knowledge of the propagation of waves in elastic media with non parallel boundaries plays its role to understand and predict the seismic behaviour at continentals margins, mountain roots, etc. Looking forward to the above concept, effect of variation of crustal thickness on Love waves were studied by different authors like De Noyer (1961), Mal (1962), Bhattacharya (1962), Sato (1952), Coulomb (1952), Stoneley (1924), Wolf (1967), Chattopadhyay (1975a), Dutta (1963a,1963b), Kar (1977).

It is noticed that seismic signals propagating through the Earth medium have to travel through various kinds of minerals which are present in the material of the Earth in the form of layers. The propagation of seismic waves is definitely effected by the elastic properties of these layered materials. Moreover, the materials of the layer might be magneto-elastic in nature. Interplay of electromagnetic field with the motion of a deformable solid has its importance owing to its theoretical and practical relevance in various branches of science and technology. Different aspects of waves in magneto-elasticity were investigated by several authors including Knopoff (1955), Dunkin \& Eringen (1963), Yu \& Tang (1966), Tomita \& Shindo (1979). A survey of linear and nonlinear wave motion in a perfectly magnetoelastic medium has been made by Bazer (1984). Othman \& Song (2006), Chattopadhyay et al (1998), Chattopadhyay \& Chowdhury (1995) investigated a few problems related to the propagation of SH wave in magneto-elastic and thermoelastic medium. An excellent review of wave motion in magnetizable deformable media will also be seen in the research papers of Maugin (1988b), Lee \& Its (1992). Hence, SH waves are always influenced by the magneto-elastic nature of media through which they have to propagate. From the literatures mentioned above it is observed that the authors did not investigate any problem where the effect of surface stress, irregularity of the surface of separation and the magneto-elastic nature of the crustal layer have been considered simultaneously, though the effect of irregular boundary and the surface stress has a prominent role in the propagation of magneto-elastic waves, specially surface waves. Motivated by the practical situation described above which the geophysicist and civil engineers have to face during the study of the seismic waves the present authors analyse the problem of the propagation of SH waves where the crustal layer is magneto-elastic with non parallel boundaries under the action of surface stress on its free surface. In our problem we have considered two types of irregularities, namely rectangular and parabolic and also comparison has been made. Variations of wave velocities due to the presence of surface stress, irregularity and the magnetic field have been studied separately. Their combined effect has also been studied. Many important peculiarities have been pointed out from the graphs. It is seen that the range of possible values of non-dimensional form of $\mathrm{SH}$ wave velocity depends on the magneto-elastic parameters. For real wave velocity two particular types of magnetic field, namely:

(i) magnetic field, acting in the direction of wave propagation and (ii) magnetic field acting in the vertical direction, transverse to the direction of wave propagation have been studied in detail. It is believed that the problem in its present form has not been investigated by any of the previous authors. 


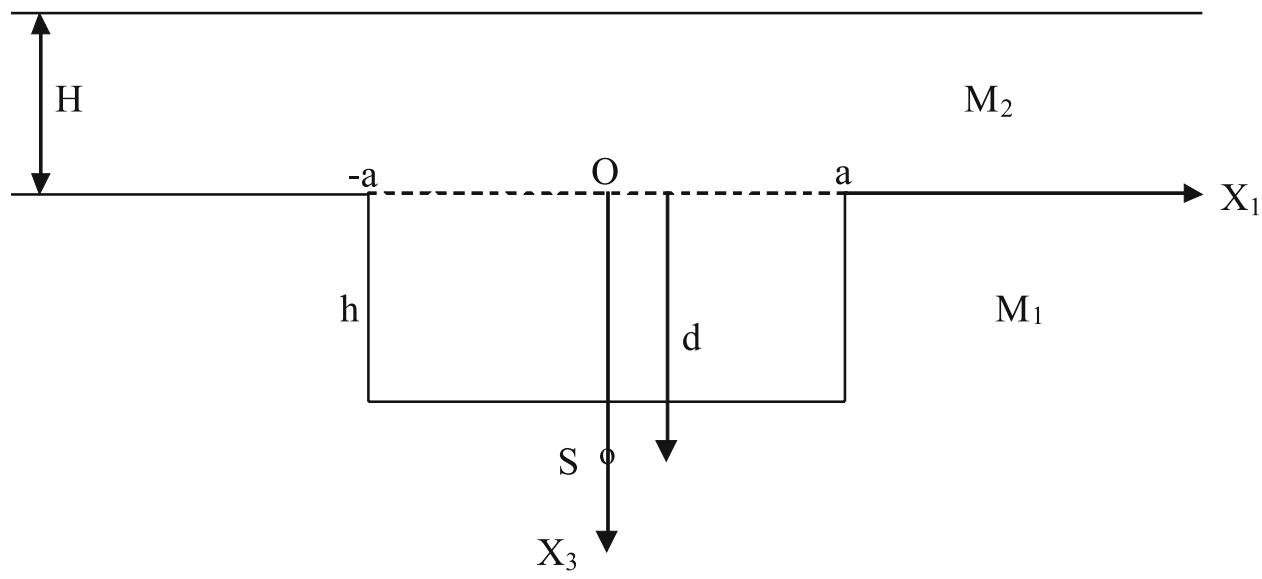

Figure 1. Schematic diagram for rectangular irregularity.

\section{Formulation of the problem}

Let us consider a model which consists of an isotropic perfectly conducting magneto-elastic crustal layer $M_{2}$ with one rectangular or parabolic irregularity on the interface between the layer and a semi infinite isotropic elastic medium $M_{1}$ as shown in the figures 1 and 2.

Let $H$ be the thickness of the layer except for the irregularities. Let us consider a rectangular system of coordinate axes $O X_{1} X_{2} X_{3}$ with the origin $O$ at the middle point of the span, $2 a$, of the irregularities. $X_{1}$ and $X_{3}$ axis are taken as shown in the figures. The positive direction of $X_{3}$-axis points vertically downwards in the medium $M_{1}$. Let us also consider that a very thin elastic continuum of two-dimensions adheres to the free surface $x_{3}=-H$ of the crustal layer. Both the media $M_{1}$ and $M_{2}$ are assumed to be homogeneous and isotropic. Let $\mathrm{S}$ be the position of the source on $X_{3}$-axis at a depth $d$ below the origin where $d>h$, in which $h$ is the depth of the rectangular irregularity held horizontally and in case of parabolic irregularity

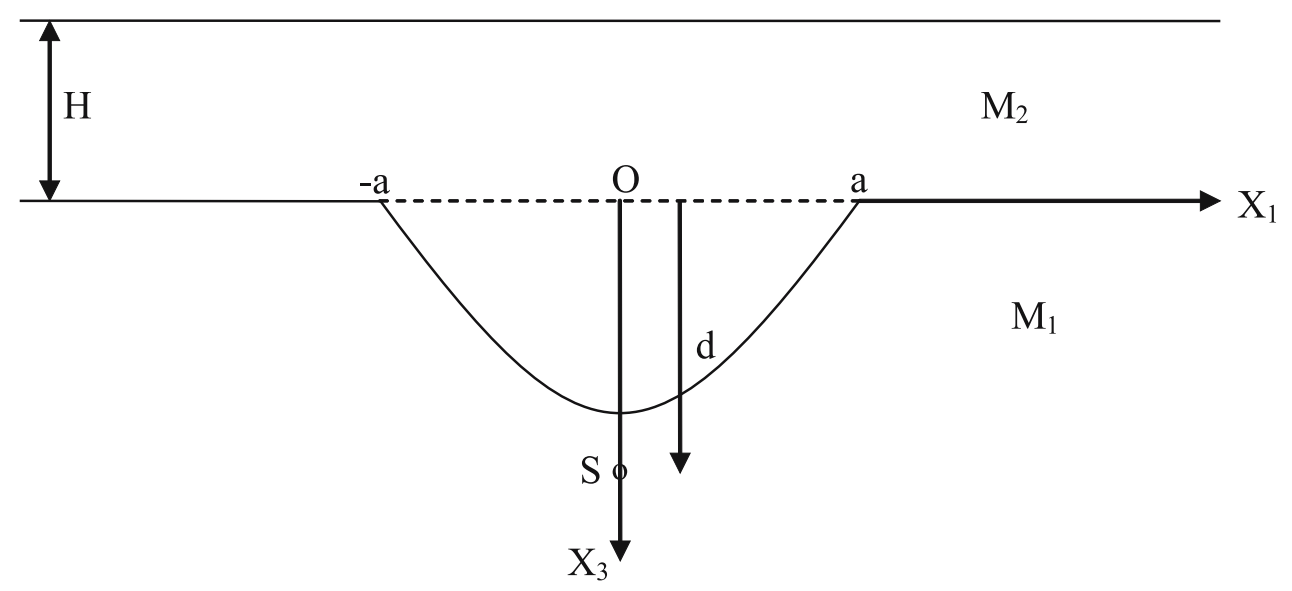

Figure 2. Schematic diagram for parabolic irregularity. 
$h$ is the depth of the vertex below $x_{3}=-H$. We assume that this source produces a time harmonic disturbance.

Thus the equations of the interfaces in the cases of rectangular and parabolic irregularities may respectively be presented as

$$
\begin{aligned}
x_{3}=\in f\left(x_{1}\right)=h & \text { for }-a \leq x_{1} \leq a \\
=0 & \text { for }\left|x_{1}\right|>a
\end{aligned}
$$

and

$$
\begin{aligned}
x_{3}=\in f\left(x_{1}\right) & =h\left(1-\frac{x_{1}^{2}}{a^{2}}\right) \quad \text { for }\left|x_{1}\right| \leq a \\
& =0 \text { for }\left|x_{1}\right|>a,
\end{aligned}
$$

where $\epsilon=\frac{h}{2 a} \ll 1$ is the perturbation parameter.

Maxwell's fundamental equations and Maxwell's stress tensor, $\left(\tau_{i j}\right)^{M}$ (Choudhary et al 2004) are respectively given by

$$
\nabla \times \boldsymbol{H}=\boldsymbol{J}, \nabla \times \boldsymbol{E}=-\frac{\partial \boldsymbol{B}}{\partial t}, \nabla \cdot \boldsymbol{B}=0, \boldsymbol{B}=\mu_{e} \boldsymbol{H}, \boldsymbol{J}=\sigma_{0}\left[\boldsymbol{E}+\frac{\partial \boldsymbol{u}}{\partial t} \times \boldsymbol{B}\right] .
$$

and

$$
\left(\tau_{i j}\right)^{M}=\mu_{e}\left(H_{i} h_{j}+H_{j} h_{i}-H_{k} h_{k} \delta_{i j}\right),
$$

where $\boldsymbol{H}=\left(H_{x_{1}}, H_{x_{2}}, H_{x_{3}}\right)$ is the total applied field, $\boldsymbol{J}$ is the electric current vector, $\boldsymbol{E}$ is the induced electric field, $\boldsymbol{B}$ is the magnetic induction vector, $\mu_{e}$ is the magnetic permeability, $\sigma_{0}$ is the electrical conductivity, $\boldsymbol{h}=\left(h_{1}, h_{2}, h_{3}\right)$ is the change in the basic magnetic field $\left(H_{1}, 0, H_{3}\right), \boldsymbol{u}$ is the displacement vector, $\delta_{i j}$ is the Kronecker delta, $t$ denotes time and $i, j, k=1,2,3$. Usual summation convention for the repeated index is applicable.

To suit the actual situation of the considered problem for the propagation of SH waves along $X_{1}$ axis one may take displacement vector as $\boldsymbol{u}=(0, v, 0)$ and all derivatives with respect to $x_{2}$ coordinate are equal to zero. In view of the above concept, taking the initial magnetic field as $\left(H_{1}, 0, H_{3}\right)$ and following (Choudhary et al 2004) one may deduce from (4) that there is no perturbation of the initial magnetic field in the $x_{1}, x_{3}$ direction and the total applied magnetic field may be presented as $H_{x_{1}}=H_{1}, H_{x_{2}}=h_{2}, H_{x_{3}}=H_{3}$ where the small perturbation $h_{2}$ in the direction of $x_{2}$ axis may be expressed as

$$
h_{2}=H_{1} \frac{\partial v}{\partial x_{1}}+H_{3} \frac{\partial v}{\partial x_{3}} .
$$

Under the present situation the Lorenz force which has only one non-zero component in the direction of $x_{2}$ axis is given by

$$
\boldsymbol{J} \times \boldsymbol{B}=\left(0, \mu_{e}\left(H_{1} \frac{\partial h_{2}}{\partial x_{1}}+H_{3} \frac{\partial h_{2}}{\partial x_{3}}\right), 0\right) .
$$

Insertion of (5) in (6) leads to

$$
(\boldsymbol{J} \times \boldsymbol{B})_{2}=\mu_{e}\left(H_{1}^{2} \frac{\partial^{2} v}{\partial x_{1}^{2}}+2 H_{1} H_{3} \frac{\partial^{2} v}{\partial x_{1} \partial x_{3}}+H_{3}^{2} \frac{\partial^{2} v}{\partial x_{3}^{2}}\right) .
$$


The dynamical equations of motion for a homogeneous isotropic perfectly conducting elastic medium $M_{2}$ having only Lorentz force may be presented as

$$
\tau_{i j, j}^{\prime}+(\boldsymbol{J} \times \boldsymbol{B})_{i}=\rho^{\prime} \frac{\partial^{2} u_{i}^{\prime}}{\partial t^{2}} \quad i, j=1,2,3,
$$

where $\tau_{i j}^{\prime}=$ components of the stress tensor $=\lambda^{\prime} e_{k k}^{\prime} \delta_{i j}+2 \mu^{\prime} e_{i j}^{\prime}$ in which $e_{i j}^{\prime}=\frac{1}{2}\left(\frac{\partial u_{i}^{\prime}}{\partial x_{j}}+\frac{\partial u_{j}^{\prime}}{\partial x_{i}}\right)$ is the components of strain tensor, $\lambda^{\prime}, \mu^{\prime}$ are Lame' elastic constants, $\rho^{\prime}$ is the density of $M_{2}, \boldsymbol{u}$ is the displacement vector such that $\boldsymbol{u}=u_{i}^{\prime}=\left(u_{1}^{\prime}, u_{2}^{\prime}, u_{3}^{\prime}\right) \cdot \tau_{i j, j}^{\prime}=\frac{\partial \tau_{i j}^{\prime}}{\partial x_{j}}, x_{j}$ denotes space coordinates of a point.

Inview of the above context the dynamical equations of motion valid for the magneto-elastic layer which is not identically satisfied may be presented as

$$
P \frac{\partial^{2} v_{2}}{\partial x_{3}^{2}}+S \frac{\partial^{2} v_{2}}{\partial x_{1} \partial x_{3}}+Q \frac{\partial^{2} v_{2}}{\partial x_{1}^{2}}=\rho^{\prime} \frac{\partial^{2} v_{2}}{\partial t^{2}},
$$

where $P=\mu^{\prime}+\mu_{e} H_{3}^{2}, S=2 \mu_{e} H_{1} H_{3}, Q=\mu^{\prime}+\mu_{e} H_{1}^{2}$, in which the displacement vector is taken as $u_{i}^{\prime}=\left(0, v_{2}, 0\right), \mu^{\prime}$ is the rigidity of the layer $M_{2}$.

For the semi-infinite elastic medium the relevant equation of motion is taken as

$$
\frac{\partial^{2} v_{1}}{\partial x_{1}^{2}}+\frac{\partial^{2} v_{1}}{\partial x_{3}^{2}}=\frac{1}{\beta_{1}^{2}} \frac{\partial^{2} v_{1}}{\partial t^{2}}
$$

where $\beta_{1}^{2}=\mu / \rho$ in which $\mu$ and $\rho$ are rigidity and density and $u_{i}=\left(0, v_{1}, 0\right)$ is the displacement vector for the medium $M_{1}$.

It is assumed that the plane $x_{3}=-H$ is a material medium adhering to the layer and is a two-dimensional elastic continuum capable of supporting its own stress represented by $\Sigma_{i \alpha}$ which obeys the law (Gurtin \& Murdoch 1976)

$$
\begin{aligned}
\Sigma_{i \alpha} & =\delta_{i \alpha}\left[\sigma+\left(\lambda_{0}+\sigma\right) u_{r, r}^{\prime}\right]+\mu_{0} u_{i, \alpha}^{\prime}+\left(\mu_{0}-\sigma\right) u_{\alpha, i}^{\prime} \quad \text { for } i, \alpha, r=1,2 \\
& =\sigma u_{3, \alpha}^{\prime} \quad \text { for } i=3,
\end{aligned}
$$

where $\lambda_{0}, \mu_{0}$ are Lame' elastic constants and $\sigma$ is the residual surface tension of material boundary $x_{3}=-H$.

Since the wave propagating in positive $x_{1}$-direction is time harmonic, we may take the solutions of (10) and (9) as $v_{j}=V_{j}\left(x_{1}, x_{3}\right) e^{i} \omega t(j=1,2 \& i=\sqrt{-1})$ and then applying Fourier transform technique in the following form

$$
\bar{V}_{1}\left(\eta, x_{3}\right)=\int_{-\infty}^{\infty} V_{1}\left(x_{1}, x_{3}\right) e^{i \eta x_{1}} d x_{1}
$$

so that

$$
V_{1}\left(x_{1}, x_{3}\right)=\frac{1}{2 \pi} \int_{-\infty}^{\infty} \bar{V}_{1}\left(\eta, x_{3}\right) e^{-i \eta x_{1}} d \eta
$$

the equations of motion finally transforms to

$$
\frac{d^{2} \bar{V}_{1}}{d x_{3}^{2}}=p_{1}^{2} \bar{V}_{1}
$$


for the medium $M_{1}$ and

$$
P \frac{d^{2} \bar{V}_{2}}{d x_{3}^{2}}-i \eta S \frac{d \bar{V}_{2}}{d x_{3}}+\left(\rho^{\prime} \omega^{2}-Q \eta^{2}\right) \bar{V}_{2}=0
$$

for the medium $M_{2}$, where $p_{1}^{2}=\eta\left(1-\frac{c^{2}}{\beta_{1}^{2}}\right)$ in which $\frac{\omega}{\eta}=c, c$ being the common wave velocity of the waves propagated along the surface.

The solutions of (13) and (14) are taken as

$$
\begin{array}{ll}
\bar{V}_{1}\left(\eta, x_{3}\right)=A e^{-p_{1} x_{3}}, & x_{3} \geq \in f\left(x_{1}\right) \\
\bar{V}_{2}\left(\eta, x_{3}\right)=B e^{p_{2} x_{3}}+C e^{p_{3} x_{3}}, & -H \leq x_{3} \leq \in f\left(x_{1}\right),
\end{array}
$$

where A,B,C are functions of $\eta$ only and $p_{2}=i \eta\left(\frac{S}{2 P}+\gamma_{2}\right), p_{3}=i \eta\left(\frac{S}{2 P}-\gamma_{2}\right)$, in which $\gamma_{2}=\sqrt{\frac{S^{2}}{4 P^{2}}+\frac{1}{P}\left(\rho^{\prime} c^{2}-Q\right)}$.

Therefore, the displacements in the medium $M_{1}$ and in the layer $M_{2}$ are given by

$$
\begin{aligned}
& V_{1}=\frac{1}{2 \pi} \int_{-\infty}^{\infty}\left(A e^{-p_{1} x_{3}}+\frac{2}{p_{1}} e^{p_{1} x_{3}} e^{-p_{1} d}\right) e^{-i \eta x_{1}} d \eta, x_{3} \geq \epsilon f\left(x_{1}\right) \\
& V_{2}=\frac{1}{2 \pi} \int_{-\infty}^{\infty}\left(B e^{p_{2} x_{3}}+C e^{p_{3} x_{3}}\right) e^{-i \eta x_{1}} d \eta,-H \leq x_{3} \leq \epsilon f\left(x_{1}\right),
\end{aligned}
$$

where the second term in the expression of $V_{1}$ is due to the effect of the source at $S$ (figures 1 and 2).

\section{Boundary conditions}

We consider the following boundary conditions:

(i) The continuity of the displacements on the interface gives rise to the condition

$$
V_{1}=V_{2} \text { on } x_{3}=\in f\left(x_{1}\right) .
$$

(ii) In view of the irregular boundary surface of separation the stress continuity condition across the interface boundary $x_{3}=\in f\left(x_{1}\right)$ may be written as

$$
\begin{gathered}
\mu \frac{\partial V_{1}}{\partial x_{3}}-\in f^{\prime}\left(x_{1}\right) \mu \frac{\partial V_{1}}{\partial x_{1}}=\left\{\mu^{\prime} \frac{\partial V_{2}}{\partial x_{3}}+\mu_{e}\left(H_{1} H_{3} \frac{\partial V_{2}}{\partial x_{1}}+H_{3}^{2} \frac{\partial V_{2}}{\partial x_{3}}\right)\right\} \\
-\in f^{\prime}\left(x_{1}\right)\left\{\mu^{\prime} \frac{\partial V_{2}}{\partial x_{1}}+\mu_{e}\left(H_{1}^{2} \frac{\partial V_{2}}{\partial x_{1}}+H_{1} H_{3} \frac{\partial V_{2}}{\partial x_{3}}\right)\right\},
\end{gathered}
$$

where Maxwell's stress tensor, given by (4) has also been taken into consideration.

(iii) Since the boundary $x_{3}=-H$ is free from external loads and surface stress acts on this boundary we have (Verma 1986, Chattopadhyay et al 1998, Gurtin \& Murdoch 1974-75)

$$
\tau_{23}^{\prime}+\tau_{23}^{M}+\sum_{2 \alpha, \alpha}-\rho_{0} \frac{\partial^{2} V_{2}}{\partial t^{2}}=0
$$


Using (4), (5) and (11) this boundary condition transforms to

$$
\mu_{0} \frac{\partial^{2} V_{2}}{\partial x_{1}^{2}}+\mu^{\prime} \frac{\partial V_{2}}{\partial x_{3}}+\mu_{e}\left(H_{1} H_{3} \frac{\partial V_{2}}{\partial x_{1}}+H_{3}^{2} \frac{\partial V_{2}}{\partial x_{3}}\right)+\rho_{0} \omega^{2} V_{2}=0 \quad \text { on } x_{3}=-H
$$

where $\rho_{0}$ is the mass per unit surface area of the geometrical surface representing the region in which surface stress is located.

\section{Solution of the problem}

From the first boundary condition we obtain

$$
\begin{gathered}
\frac{1}{2 \pi} \int_{-\infty}^{\infty}\left(A e^{-p_{1} \in f\left(x_{1}\right)}+\frac{2}{p_{1}} e^{p_{1} \in f\left(x_{1}\right)} e^{-p_{1} d}\right) e^{-i \eta x_{1}} d \eta \\
=\frac{1}{2 \pi} \int_{-\infty}^{\infty}\left(B e^{p_{2} \in f\left(x_{1}\right)}+C e^{p_{3} \in f\left(x_{1}\right)}\right) e^{-i \eta x_{1}} d \eta
\end{gathered}
$$

Expanding $A, B, C, e^{ \pm p_{r} \in f\left(x_{1}\right)}$ in powers of $\in$ and since $\in$ is very small, we get

$$
\begin{aligned}
& \in \int_{-\infty}^{\infty}\left(p_{1} A_{0}+p_{2} B_{0}+p_{3} C_{0}-2 e^{-p_{1} d}\right) f\left(x_{1}\right) e^{-i \eta x_{1}} d \eta \\
& \quad=\int_{-\infty}^{\infty}\left[\left(A_{0}-B_{0}-C_{0}\right)+\in\left(A_{1}-B_{1}-C_{1}\right)+\frac{2}{p_{1}} e^{-p_{1} d}\right] e^{-i \eta x_{1}} d \eta
\end{aligned}
$$

Similarly, from the second boundary condition, one obtains the following

$$
\begin{aligned}
\in \int_{-\infty}^{\infty} & {\left[\left\{\mu p_{1}^{2} A_{0}-\mu^{\prime} p_{2}^{2} B_{0}-\mu^{\prime} p_{3}^{2} C_{0}+2 \mu p_{1} e^{-p_{1} d}-\mu_{e} H_{3}^{2} p_{2}^{2} B_{0}\right.\right.} \\
& \left.-\mu_{e} H_{3}^{2} p_{3}^{2} C_{0}+i \eta \mu_{e} H_{1} H_{3} p_{2} B_{0}+i \eta \mu_{e} H_{1} H_{3} p_{3} C_{0}\right\} f\left(x_{1}\right) \\
& +\left\{\mu A_{0}-\mu^{\prime} B_{0}-\mu^{\prime} C_{0}+\frac{2 \mu}{p_{1}} e^{-p_{1} d}-\frac{i \mu_{e} H_{1} H_{3} p_{2} B_{0}}{\eta}\right. \\
& \left.\left.-\frac{i \mu_{e} H_{1} H_{3} p_{3} C_{0}}{\eta}-\mu_{e} H_{1}^{2} B_{0}-\mu_{e} H_{1}^{2} C_{0}\right\} i \eta f^{\prime}\left(x_{1}\right)\right] e^{-i \eta x_{1}} d \eta \\
= & \int_{-\infty}^{\infty}\left[\mu p_{1} A_{0}+\mu^{\prime} p_{2} B_{0}+\mu^{\prime} p_{3} C_{0}-2 \mu e^{-p_{1} d}+\mu_{e} H_{3}^{2} p_{2} B_{0}\right. \\
& +\mu_{e} H_{3}^{2} p_{3} C_{0}-i \eta \mu_{e} H_{1} H_{3} B_{0}-i \eta \mu_{e} H_{1} H_{3} C_{0}+\in\left\{\mu p_{1} A_{1}+\mu^{\prime} p_{2} B_{1}\right. \\
& +\mu^{\prime} p_{3} C_{1}+\mu_{e} H_{3}^{2} p_{2} B_{1}+\mu_{e} H_{3}^{2} p_{3} C_{1}-i \eta \mu_{e} H_{1} H_{3} B_{1} \\
& \left.\left.-i \eta \mu_{e} H_{1} H_{3} C_{1}\right\}\right] e^{-i \eta x_{1}} d \eta
\end{aligned}
$$


Let us apply Fourier transform on $f\left(x_{1}\right)$ as

$$
\bar{f}(\xi)=\int_{-\infty}^{\infty} f\left(x_{1}\right) e^{i \xi x_{1}} d x_{1}
$$

so that

$$
f\left(x_{1}\right)=\frac{1}{2 \pi} \int_{-\infty}^{\infty} \bar{f}(\xi) e^{-i \xi x_{1}} d \xi
$$

and

$$
f^{\prime}\left(x_{1}\right)=-\frac{i}{2 \pi} \int_{-\infty}^{\infty} \xi \bar{f}(\xi) e^{-i \xi x_{1}} d \xi
$$

We now apply transform (20) on (18) and (19) and get

$$
\begin{array}{r}
\frac{\epsilon}{2 \pi} \int_{-\infty}^{\infty}\left\{\int_{-\infty}^{\infty}\left(p_{1} A_{0}+p_{2} B_{0}+p_{3} C_{0}-2 e^{-p_{1} d}\right) e^{-i(\eta+\xi) x_{1}} d \eta\right\} \bar{f}(\xi) d \xi \\
\quad=\int_{-\infty}^{\infty}\left[A_{0}-B_{0}-C_{0}+\in\left(A_{1}-B_{1}-C_{1}\right)+\frac{2}{p_{1}} e^{-p_{1} d}\right] e^{-i \eta x_{1}} d \eta
\end{array}
$$

and

$$
\begin{aligned}
\frac{\epsilon}{2 \pi} \int_{-\infty}^{\infty}\left\{\int _ { - \infty } ^ { \infty } \left(\mu p_{1}^{2} A_{0}-\mu^{\prime} p_{2}^{2} B_{0}-\mu^{\prime} p_{3}^{2} C_{0}+2 \mu p_{1} e^{-p_{1} d}-\mu_{e} H_{3}^{2} p_{2}^{2} B_{0}\right.\right. \\
\left.\left.\quad-\mu_{e} H_{3}^{2} p_{3}^{2} C_{0}+i \eta \mu_{e} H_{1} H_{3} p_{2} B_{0}+i \eta \mu_{e} H_{1} H_{3} p_{3} C_{0}\right) e^{-i(\eta+\xi) x_{1}} d \eta\right\} \bar{f}(\xi) d \xi \\
\quad+\frac{\epsilon}{2 \pi} \int_{-\infty}^{\infty}\left\{\int _ { - \infty } ^ { \infty } \left(\mu A_{0}-\mu^{\prime} B_{0}-\mu^{\prime} C_{0}+\frac{2 \mu}{p_{1}} e^{-p_{1} d}-\frac{i \mu_{e} H_{1} H_{3} p_{2} B_{0}}{\eta}\right.\right. \\
\left.\left.\quad-\frac{i \mu_{e} H_{1} H_{3} p_{3} C_{0}}{\eta}-\mu_{e} H_{1}^{2} B_{0}-\mu_{e} H_{1}^{2} C_{0}\right) e^{-i(\eta+\xi) x_{1}} \eta d \eta\right\} \xi \bar{f}(\xi) d \xi \\
=\int_{-\infty}^{\infty}\left\{\mu p_{1} A_{0}+\mu^{\prime} p_{2} B_{0}+\mu^{\prime} p_{3} C_{0}-2 \mu e^{-p_{1} d}+\mu_{e} H_{3}^{2} p_{2} B_{0}+\mu_{e} H_{3}^{2} p_{3} C_{0}\right. \\
\quad-i \eta \mu_{e} H_{1} H_{3} B_{0}-i \eta \mu_{e} H_{1} H_{3} C_{0}+\in\left(\mu p_{1} A_{1}+\mu^{\prime} p_{2} B_{1}+\mu^{\prime} p_{3} C_{1}\right. \\
\left.\left.\quad+\mu_{e} H_{3}^{2} p_{2} B_{1}+\mu_{e} H_{3}^{2} p_{3} C_{1}-i \eta \mu_{e} H_{1} H_{3} B_{1}-i \eta \mu_{e} H_{1} H_{3} C_{1}\right)\right\} e^{-i \eta x_{1}} d \eta
\end{aligned}
$$

Taking $\eta+\xi=\kappa$ where $\xi$ is constant such that $d \eta=d \kappa$ and solving the inner integral of LHS of (21) and (22) and replacing $\eta$ in the RHS by $\kappa$ we get

$$
\in \int_{-\infty}^{\infty} R_{1}(\kappa) e^{-i \kappa x_{1}} d \kappa=\int_{-\infty}^{\infty}\left\{A_{0}-B_{0}-C_{0}+\in\left(A_{1}-B_{1}-C_{1}\right)+\frac{2}{p_{1}} e^{-p_{1} d}\right\} e^{-i \kappa x_{1}} d \kappa
$$


or,

$$
A_{0}-B_{0}-C_{0}+\in\left(A_{1}-B_{1}-C_{1}\right)+\frac{2}{p_{1}} e^{-p_{1} d}=\in R_{1}(\kappa)
$$

and

$$
\begin{aligned}
& \in \int_{-\infty}^{\infty} R_{2}(\kappa) e^{-i \kappa x_{1}} d \kappa=\int_{-\infty}^{\infty}\left\{\mu p_{1} A_{0}+\mu^{\prime} p_{2} B_{0}+\mu^{\prime} p_{3} C_{0}-2 \mu e^{-p_{1} d}\right. \\
& \quad+\mu_{e} H_{3}^{2} p_{2} B_{0}+\mu_{e} H_{3}^{2} p_{3} C_{0}-i \eta \mu_{e} H_{1} H_{3} B_{0}-i \eta \mu_{e} H_{1} H_{3} C_{0} \\
& \quad+\in\left(\mu p_{1} A_{1}+\mu^{\prime} p_{2} B_{1}+\mu^{\prime} p_{3} C_{1}+\mu_{e} H_{3}^{2} p_{2} B_{1}+\mu_{e} H_{3}^{2} p_{3} C_{1}\right. \\
& \left.\left.\quad-i \eta \mu_{e} H_{1} H_{3} B_{1}-i \eta \mu_{e} H_{1} H_{3} C_{1}\right)\right\} e^{-i \kappa x_{1}} d \kappa
\end{aligned}
$$

or,

$$
\begin{gathered}
\mu p_{1} A_{0}+\mu^{\prime} p_{2} B_{0}+\mu^{\prime} p_{3} C_{0}+\mu_{e} H_{3}^{2} p_{2} B_{0}+\mu_{e} H_{3}^{2} p_{3} C_{0}-i \eta \mu_{e} H_{1} H_{3} B_{0} \\
-i \eta \mu_{e} H_{1} H_{3} C_{0}+\in\left(\mu p_{1} A_{1}+\mu^{\prime} p_{2} B_{1}+\mu^{\prime} p_{3} C_{1}+\mu_{e} H_{3}^{2} p_{2} B_{1}\right. \\
\left.+\mu_{e} H_{3}^{2} p_{3} C_{1}-i \eta \mu_{e} H_{1} H_{3} B_{1}-i \eta \mu_{e} H_{1} H_{3} C_{1}\right)-2 \mu e^{-p_{1} d}=\in R_{2}(\kappa)
\end{gathered}
$$

where

$$
R_{1}(\kappa)=\frac{1}{2 \pi} \int_{-\infty}^{\infty}\left[p_{1} A_{0}+p_{2} B_{0}+p_{3} C_{0}-2 e^{-p_{1} d}\right]_{\eta=\kappa-\xi} \bar{f}(\xi) d \xi
$$

and

$$
\begin{aligned}
R_{2}(\kappa)= & \frac{1}{2 \pi} \int_{-\infty}^{\infty}\left[\mu p_{1}^{2} A_{0}-\mu^{\prime} p_{2}^{2} B_{0}-\mu^{\prime} p_{3}^{2} C_{0}+2 \mu p_{1} e^{-p_{1} d}-\mu_{e} H_{3}^{2} p_{2}^{2} B_{0}\right. \\
& -\mu_{e} H_{3}^{2} p_{3}^{2} C_{0}+i \eta \mu_{e} H_{1} H_{3} p_{2} B_{0}+i \eta \mu_{e} H_{1} H_{3} p_{3} C_{0} \\
& +\left(\mu A_{0}-\mu^{\prime} B_{0}-\mu^{\prime} C_{0}+\frac{2 \mu}{p_{1}} e^{-p_{1} d}-\frac{i \mu_{e} H_{1} H_{3} p_{2} B_{0}}{\eta}\right. \\
& \left.\left.-\frac{i \mu_{e} H_{1} H_{3} p_{3} C_{0}}{\eta}-\mu_{e} H_{1}^{2} B_{0}-\mu_{e} H_{1}^{2} C_{0}\right) \xi \eta\right]_{\eta=\kappa-\xi} \bar{f}(\xi) d \xi
\end{aligned}
$$

From the third boundary condition we have

$$
\begin{aligned}
& \left(-\mu^{\prime} p_{2}+\mu_{0} \eta^{2}-\rho_{0} \omega^{2}-\mu_{e} H_{3}^{2} p_{2}+i \eta \mu_{e} H_{1} H_{3}\right) e^{-p_{2} H} B_{0}-\left(\mu^{\prime} p_{3}-\mu_{0} \eta^{2}\right. \\
& \left.\quad+\rho_{0} \omega^{2}+\mu_{e} H_{3}^{2} p_{3}-i \eta \mu_{e} H_{1} H_{3}\right) e^{-p_{3} H} C_{0}+\in\left[\left(-\mu^{\prime} p_{2}+\mu_{0} \eta^{2}\right.\right. \\
& \left.\quad-\rho_{0} \omega^{2}-\mu_{e} H_{3}^{2} p_{2}+i \eta \mu_{e} H_{1} H_{3}\right) e^{-p_{2} H} B_{1}-\left(\mu^{\prime} p_{3}-\mu_{0} \eta^{2}+\rho_{0} \omega^{2}\right. \\
& \left.\left.\quad+\mu_{e} H_{3}^{2} p_{3}-i \eta \mu_{e} H_{1} H_{3}\right) e^{-p_{3} H} C_{1}\right]=0
\end{aligned}
$$


Equating absolute terms and coefficients of $\in$ from (23), (25) and (27) one obtains the following

$$
\begin{aligned}
& A_{0}-B_{0}-C_{0}+\frac{2}{p_{1}} e^{-p_{1} d}=0 \\
& \mu p_{1} A_{0}+\mu^{\prime} p_{2} B_{0}+\mu^{\prime} p_{3} C_{0}+\mu_{e} H_{3}^{2} p_{2} B_{0}+\mu_{e} H_{3}^{2} p_{3} C_{0} \\
& \quad-i \eta \mu_{e} H_{1} H_{3} B_{0}-i \eta \mu_{e} H_{1} H_{3} C_{0}-2 \mu e^{-p_{1} d}=0 \\
& \left(-\mu^{\prime}-\mu_{e} H_{3}^{2}\right)\left(p_{2} e^{-p_{2} H} B_{0}+p_{3} e^{-p_{3} H} C_{0}\right) \\
& \quad+\left(\mu_{0} \eta^{2}-\rho_{0} \omega^{2}+i \eta \mu_{e} H_{1} H_{3}\right)\left(e^{-p_{2} H} B_{0}+e^{-p_{3} H} C_{0}\right)=0 \\
& A_{1}-B_{1}-C_{1}-R_{1}(\kappa)=0 \\
& \mu p_{1} A_{1}+\mu^{\prime} p_{2} B_{1}+\mu^{\prime} p_{3} C_{1}+\mu_{e} H_{3}^{2} p_{2} B_{1}+\mu_{e} H_{3}^{2} p_{3} C_{1} \\
& \quad-i \eta \mu_{e} H_{1} H_{3} B_{1}-i \eta \mu_{e} H_{1} H_{3} C_{1}-R_{2}(\kappa)=0 \\
& \left(-\mu^{\prime}-\mu_{e} H_{3}^{2}\right)\left(p_{2} e^{-p_{2} H} B_{1}+p_{3} e^{-p_{3} H} C_{1}\right) \\
& \quad+\left(\mu_{0} \eta^{2}-\rho_{0} \omega^{2}+i \eta \mu_{e} H_{1} H_{3}\right)\left(e^{-p_{2} H} B_{1}+e^{-p_{3} H} C_{1}\right)=0
\end{aligned}
$$

Solving (28)-(33), we get

$$
\begin{aligned}
& A_{0}=4 \mu e^{-p_{1} d} \frac{R_{3}+\left(\mu_{0} \eta^{2}-\rho_{0} \omega^{2}+i \eta \mu_{e} H_{1} H_{3}\right)\left(e^{-p_{2} H}-e^{-p_{3} H}\right)}{\bar{R}(\kappa)}-\frac{2}{p_{1}} e^{-p_{1} d} \\
& B_{0}=4 \mu e^{-p_{1} d} \frac{\mu^{\prime} p_{3}-\left(\mu_{0} \eta^{2}-\rho_{0} \omega^{2}\right)+\mu_{e} H_{3}^{2} p_{3}-i \eta \mu_{e} H_{1} H_{3}}{\bar{R}(\kappa)} e^{-p_{3} H} \\
& C_{0}=4 \mu e^{-p_{1} d} \frac{-\mu^{\prime} p_{2}+\left(\mu_{0} \eta^{2}-\rho_{0} \omega^{2}\right)-\mu_{e} H_{3}^{2} p_{2}+i \eta \mu_{e} H_{1} H_{3}}{\bar{R}(\kappa)} e^{-p_{2} H} \\
& A_{1}=\left\{R_{2}(\kappa)-\mu p_{1} R_{1}(\kappa)\right\} \frac{R_{3}+\left(\mu_{0} \eta^{2}-\rho_{0} \omega^{2}+i \eta \mu_{e} H_{1} H_{3}\right)\left(e^{-p_{2} H}-e^{-p_{3} H}\right)}{\bar{R}(\kappa)}+R_{1}(\kappa) \\
& B_{1}=\left\{R_{2}(\kappa)-\mu p_{1} R_{1}(\kappa)\right\} \frac{\mu^{\prime} p_{3}-\left(\mu_{0} \eta^{2}-\rho_{0} \omega^{2}\right)+\mu_{e} H_{3}^{2} p_{3}-i \eta \mu_{e} H_{1} H_{3}}{\bar{R}(\kappa)} e^{-p_{3} H} \\
& C_{1}=\left\{R_{2}(\kappa)-\mu p_{1} R_{1}(\kappa)\right\} \frac{-\mu^{\prime} p_{2}+\left(\mu_{0} \eta^{2}-\rho_{0} \omega^{2}\right)-\mu_{e} H_{3}^{2} p_{2}+i \eta \mu_{e} H_{1} H_{3}}{\bar{R}(\kappa)} e^{-p_{2} H}
\end{aligned}
$$


where

$$
\begin{aligned}
\bar{R}(\kappa)= & \left\{\mu^{\prime} p_{3}-\left(\mu_{0} \eta^{2}-\rho_{0} \omega^{2}\right)+\mu_{e} H_{3}^{2} p_{3}-i \eta \mu_{e} H_{1} H_{3}\right\} \\
& \left(\mu p_{1}+\mu^{\prime} p_{2}+\mu_{e} H_{3}^{2} p_{2}-i \eta \mu_{e} H_{1} H_{3}\right) e^{-p_{3} H} \\
& +\left\{-\mu^{\prime} p_{2}+\left(\mu_{0} \eta^{2}-\rho_{0} \omega^{2}\right)-\mu_{e} H_{3}^{2} p_{2}+i \eta \mu_{e} H_{1} H_{3}\right\} \\
& \left(\mu p_{1}+\mu^{\prime} p_{3}+\mu_{e} H_{3}^{2} p_{3}-i \eta \mu_{e} H_{1} H_{3}\right) e^{-p_{2} H}
\end{aligned}
$$

and $R_{3}=\left(-\mu^{\prime}-\mu_{e} H_{3}^{2}\right)\left(p_{2} e^{-p_{2} H}-p_{3} e^{-p_{3} H}\right)$.

The displacement in the layer is

$$
\begin{aligned}
V_{2}\left(x_{1}, x_{3}\right)= & \frac{1}{2 \pi} \int_{-\infty}^{\infty}\left(B e^{p_{2} x_{3}}+C e^{p_{3} x_{3}}\right) e^{-i \eta x_{1}} d \eta \\
= & \frac{1}{2 \pi} \int_{-\infty}^{\infty}\left\{\left(e^{p_{2} x_{3}} B_{0}+e^{p_{3} x_{3}} C_{0}\right)+\in\left(e^{p_{2} x_{3}} B_{1}+e^{p_{3} x_{3}} C_{1}\right)\right\} e^{-i \eta x_{1}} d \eta \\
= & \frac{1}{2 \pi} \int_{-\infty}^{\infty} \frac{4 \mu}{\bar{R}(\kappa)} e^{-p_{1} d}\left[1+\epsilon \frac{\left\{R_{2}(\kappa)-\mu p_{1} R_{1}(\kappa)\right\}}{4 \mu} e^{p_{1} d}\right] \\
& \times\left[\left\{\mu^{\prime} p_{3}-\left(\mu_{0} \eta^{2}-\rho_{0} \omega^{2}\right)+\mu_{e} H_{3}^{2} p_{3}-i \eta \mu_{e} H_{1} H_{3}\right\} e^{p_{2} x_{3}-p_{3} H}\right. \\
& \left.+\left\{-\mu^{\prime} p_{2}+\left(\mu_{0} \eta^{2}-\rho_{0} \omega^{2}\right)-\mu_{e} H_{3}^{2} p_{2}+i \eta \mu_{e} H_{1} H_{3}\right\} e^{p_{3} x_{3}-p_{2} H}\right] e^{-i \eta x_{1}} d \eta
\end{aligned}
$$

Now $\left\{R_{2}(\kappa)-\mu p_{1} R_{1}(\kappa)\right\}$

$$
\begin{aligned}
= & \frac{1}{2 \pi} \int_{-\infty}^{\infty}\left[\left(\mu p_{1}^{2} A_{0}-\mu^{\prime} p_{2}^{2} B_{0}-\mu^{\prime} p_{3}^{2} C_{0}+2 \mu p_{1} e^{-p_{1} d}-\mu_{e} H_{3}^{2} p_{2}^{2} B_{0}\right.\right. \\
& \left.-\mu_{e} H_{3}^{2} p_{3}^{2} C_{0}+i \eta \mu_{e} H_{1} H_{3} p_{2} B_{0}+i \eta \mu_{e} H_{1} H_{3} p_{3} C_{0}\right) \\
& +\left(\mu A_{0}-\mu^{\prime} B_{0}-\mu^{\prime} C_{0}+\frac{2 \mu}{p_{1}} e^{-p_{1} d}-\frac{i \mu_{e} H_{1} H_{3} p_{2}}{\eta} B_{0}\right. \\
& \left.\left.-\frac{i \mu_{e} H_{1} H_{3} p_{3}}{\eta} C_{0}-\mu_{e} H_{1}^{2} B_{0}-\mu_{e} H_{1}^{2} C_{0}\right)\right]_{\eta=\kappa-\xi} \bar{f}(\xi) d \xi \\
& -\frac{1}{2 \pi} \int_{-\infty}^{\infty} \mu p_{1}\left[p_{1} A_{0}+p_{2} B_{0}+p_{3} C_{0}-2 e^{-p_{1} d}\right]_{\eta=\kappa-\xi} \bar{f}(\xi) d \xi \\
= & \frac{2 \mu}{\pi} \int_{-\infty}^{\infty}\left[-p_{1} p_{2}\left(\mu-\mu^{\prime}-\mu_{e} H_{3}^{2}\right) M e^{-p_{3} H}\right. \\
& -p_{1} p_{3}\left(\mu-\mu^{\prime}-\mu_{e} H_{3}^{2}\right) N e^{-p_{2} H}-\left\{\mu^{\prime} p_{2}^{2}-\mu p_{1}^{2}+\left(\mu^{\prime}-\mu+\mu_{e} H_{1}^{2}\right) \xi \eta\right.
\end{aligned}
$$




$$
\begin{aligned}
& \left.+\mu_{e} H_{3}^{2} p_{2}^{2}-i \eta \mu_{e} H_{1} H_{3} p_{2}+i \eta \mu_{e} H_{1} H_{3} p_{1}+i \mu_{e} H_{1} H_{3} p_{2} \xi\right\} M e^{-p_{3} H} \\
& -\left\{\mu^{\prime} p_{3}^{2}-\mu p_{1}^{2}+\left(\mu^{\prime}-\mu+\mu_{e} H_{1}^{2}\right) \xi \eta+\mu_{e} H_{3}^{2} p_{3}^{2}-i \eta \mu_{e} H_{1} H_{3} p_{3}\right. \\
& \left.\left.\left.+i \eta \mu_{e} H_{1} H_{3} p_{1}+i \mu_{e} H_{1} H_{3} p_{3} \xi\right\} N e^{-p_{2} H}\right] \frac{e^{-p_{1} d}}{\bar{R}(\kappa)}\right]_{\eta=\kappa-\xi} \bar{f}(\xi) d \xi
\end{aligned}
$$

where

$$
\begin{aligned}
& M=\mu^{\prime} p_{3}-\left(\mu_{0} \eta^{2}-\rho_{0} \omega^{2}\right)+\mu_{e} H_{3}^{2} p_{3}-i \eta \mu_{e} H_{1} H_{3} \\
& N=-\mu^{\prime} p_{2}+\left(\mu_{0} \eta^{2}-\rho_{0} \omega^{2}\right)-\mu_{e} H_{3}^{2} p_{2}+i \eta \mu_{e} H_{1} H_{3}
\end{aligned}
$$

\subsection{Rectangular irregularity}

From (1) the interface for the rectangular irregularity is

$$
\bar{f}(\xi)=\frac{4 a}{\xi} \sin \xi a
$$

Hence,

$$
R_{2}(\kappa)-\mu p_{1} R_{1}(\kappa)=\frac{8 \mu a}{\pi} \int_{-\infty}^{\infty}[g(\kappa-\xi)+g(\kappa+\xi)] \frac{\sin \xi a}{\xi} d \xi
$$

where

$$
\begin{aligned}
g(\kappa- & \xi) \\
= & {\left[\left[-p_{1} p_{2}\left(\mu-\mu^{\prime}-\mu_{e} H_{3}^{2}\right) M e^{-p_{3} H}-p_{1} p_{3}\left(\mu-\mu^{\prime}-\mu_{e} H_{3}^{2}\right) N e^{-p_{2} H}\right.\right.} \\
& -\left\{\mu^{\prime} p_{2}^{2}-\mu p_{1}^{2}+\left(\mu^{\prime}-\mu+\mu_{e} H_{1}^{2}\right) \xi \eta+\mu_{e} H_{3}^{2} p_{2}^{2}-i \eta \mu_{e} H_{1} H_{3} p_{2}\right. \\
& \left.+i \eta \mu_{e} H_{1} H_{3} p_{1}+i \mu_{e} H_{1} H_{3} p_{2} \xi\right\} M e^{-p_{3} H}-\left\{\mu^{\prime} p_{3}^{2}-\mu p_{1}^{2}\right. \\
& +\left(\mu^{\prime}-\mu+\mu_{e} H_{1}^{2}\right) \xi \eta+\mu_{e} H_{3}^{2} p_{3}^{2}-i \eta \mu_{e} H_{1} H_{3} p_{3}+i \eta \mu_{e} H_{1} H_{3} p_{1} \\
& \left.\left.\left.+i \mu_{e} H_{1} H_{3} p_{3} \xi\right\} N e^{-p_{2} H}\right] \frac{e^{-p_{1} d}}{\bar{R}(\kappa)}\right]_{\eta=\kappa-\xi}
\end{aligned}
$$

Following the asymptotic formula of Willis (1948) and Tranter (1966) we get

$$
\int_{-\infty}^{\infty}[g(\kappa-\xi)+g(\kappa+\xi)] \frac{\sin \xi a}{\xi} d \xi=\pi g(\kappa) .
$$

Hence from (37) one obtains $\in \frac{R_{2}(\kappa)-\mu p_{1} R_{1}(\kappa)}{4 \mu}=h g(\kappa)$. 
Now the displacement in $M_{2}$ is

$$
\begin{aligned}
V_{2}\left(x_{1}, x_{3}\right) & =\frac{1}{2 \pi} \int_{-\infty}^{\infty} \frac{4 \mu}{\bar{R}(\kappa)} e^{-p_{1} d}\left[1+h g(\kappa) e^{p_{1} d}\right]\left[M e^{p_{2} x_{3}-p_{3} H}+N e^{p_{3} x_{3}-p_{2} H}\right] e^{-i \eta x_{1}} d \xi \\
& =\frac{1}{2 \pi} \int_{-\infty}^{\infty} \frac{4 \mu e^{-p_{1} d}\left[M e^{p_{2} x_{3}-p_{3} H}+N e^{p_{3} x_{3}-p_{2} H}\right]}{\bar{R}(\kappa)\left[1-h g(\kappa) e^{p_{1} d}\right]} e^{-i \eta x_{1}} d \eta .
\end{aligned}
$$

The dispersion equation is

$$
\bar{R}(\kappa)\left[1-h g(\kappa) e^{p_{1} d}\right]=0,
$$

which on substitution of the expressions for $\bar{R}(\kappa)$ and $g(\kappa)$ transforms to

$$
\begin{aligned}
\left(\mu p_{1}\right. & \left.+\mu^{\prime} p_{2}+\mu_{e} H_{3}^{2} p_{2}-i \eta \mu_{e} H_{1} H_{3}\right) M e^{-p_{3} H} \\
& +\left(\mu p_{1}+\mu^{\prime} p_{3}+\mu_{e} H_{3}^{2} p_{3}-i \eta \mu_{e} H_{1} H_{3}\right) N e^{-p_{2} H} \\
& -h\left[-p_{1} p_{2}\left(\mu-\mu^{\prime}-\mu_{e} H_{3}^{2}\right) M e^{-p_{3} H}-p_{1} p_{3}\left(\mu-\mu^{\prime}-\mu_{e} H_{3}^{2}\right) N e^{-p_{2} H}\right. \\
& -\left(\mu^{\prime} p_{2}^{2}-\mu p_{1}^{2}+\mu_{e} H_{3}^{2} p_{2}^{2}-i \eta \mu_{e} H_{1} H_{3} p_{2}+i \eta \mu_{e} H_{1} H_{3} p_{1}\right) M e^{-p_{3} H} \\
& \left.-\left(\mu^{\prime} p_{3}^{2}-\mu p_{1}^{2}+\mu_{e} H_{3}^{2} p_{3}^{2}-i \eta \mu_{e} H_{1} H_{3} p_{3}+i \eta \mu_{e} H_{1} H_{3} p_{1}\right) N e^{-p_{2} H}\right]=0 .
\end{aligned}
$$

After modification the equation (39) takes the following form

$$
\tan \eta H \Gamma=\frac{N_{0}}{D_{0}}
$$

where

$$
\begin{aligned}
\Gamma=\frac{R_{a}}{R_{b}^{2}} c_{b}, N_{0}= & \Gamma\left[R_{b}^{2}\left\{\frac{\mu c_{a}}{\mu^{\prime}}-h \eta\left(\frac{\mu c_{a}^{2}}{\mu^{\prime}}+\frac{R_{a}^{2} c_{b}^{2}}{R_{b}^{2}}\right)\right\}\right. \\
& \left.+\frac{\eta}{k_{0}}\left(\eta^{\prime 2}-\frac{c^{2}}{\beta_{2}^{2}}\right)\left\{R_{b}^{2}+h \eta c_{a}\left(\frac{\mu}{\mu^{\prime}}-R_{b}^{2}\right)\right\}\right]
\end{aligned}
$$

and

$$
\begin{aligned}
D_{0}= & \frac{R_{a}^{2} c_{b}^{2}}{R_{b}^{4}}\left\{R_{b}^{4}+h \eta c_{a}\left(\frac{\mu R_{b}^{2}}{\mu^{\prime}}-R_{b}^{4}\right)\right\} \\
& -\frac{\eta}{k_{0}}\left(\eta^{\prime 2}-\frac{c^{2}}{\beta_{2}^{2}}\right)\left\{\frac{\mu c_{a}}{\mu^{\prime}}-h \eta\left(\frac{\mu c_{a}^{2}}{\mu^{\prime}}+\frac{R_{a}^{2} c_{b}^{2}}{R_{b}^{2}}\right)\right\}
\end{aligned}
$$

in which

$$
\begin{aligned}
& R_{a}^{2}=1+\frac{\mu_{e} H_{1}^{2}}{\mu^{\prime}}+\frac{\mu_{e} H_{3}^{2}}{\mu^{\prime}}, R_{b}^{2}=1+\frac{\mu_{e} H_{3}^{2}}{\mu^{\prime}}, c_{b}^{2}=\frac{c^{2}}{\beta_{2}^{2}}-1, \\
& c_{a}^{2}=1-\frac{c^{2}}{\beta_{1}^{2}}, k_{0}=\frac{\mu^{\prime}}{\rho_{0} \beta_{2}^{2}}, \beta_{2}^{2}=\frac{\mu^{\prime} R_{a}^{2}}{\rho^{\prime} R_{b}^{2}}, \eta^{\prime 2}=\frac{\mu_{0}}{\rho_{0} \beta_{2}^{2}} .
\end{aligned}
$$


If the interface is regular (i.e. $h=0$ ), but the free surface of the crustal layer is acted on by the surface stress, the corresponding dispersion equation is deduced from (40) as

$$
\tan \eta H \Gamma=\frac{N_{1}}{D_{1}}
$$

When the surface stress is absent (i.e. $\eta^{\prime 2}=c^{2} / \beta_{2}^{2}$ ) but the interface is irregular of rectangular type, the concerned dispersion equation is obtained from (40) as

$$
\tan \eta H \Gamma=\frac{N_{2}}{D_{2}} .
$$

In the above the expressions for $N_{1}, D_{1}$ and $N_{2}, D_{2}$ are deduced from $N_{0}, D_{0}$ of (40) by putting $h=0$ and $\eta^{\prime 2}=c^{2} / \beta_{2}^{2}$ respectively.

When the interface is regular and $x_{3}=-H$ is free of surface stress then equation (40) reduces to the dispersion relation for the plane $\mathrm{SH}$ waves in a magneto-elastic crustal layer lying over an isotropic solid elastic half space as given below

$$
\tan \eta H \Gamma=\frac{\mu c_{a}}{\mu^{\prime} R_{a} c_{b}} .
$$

\subsection{Parabolic irregularity}

Applying Fourier transform to the equation (2), the interface is

$$
\bar{f}(\xi)=\frac{4 h a}{\epsilon} \times \frac{\sin \xi a-a \xi \cos \xi a}{(\xi a)^{3}} .
$$

Now,

$$
\begin{aligned}
R_{2}(\kappa)-\mu p_{1} R_{1}(\kappa) & =\frac{2 \mu}{\pi} \int_{0}^{\infty}[g(\kappa-\xi)+g(\kappa+\xi)] \bar{f}(\xi) d \xi \\
& =\frac{8 a h \mu}{\pi \in} \int_{0}^{\infty}[g(\kappa-\xi)+g(\kappa+\xi)] \sqrt{\frac{\pi}{2}} \frac{J_{3 / 2}(\xi a)}{(\xi a)^{\frac{3}{2}}} d \xi \\
& =\frac{16 h \mu}{3 \pi \epsilon} g(\kappa),
\end{aligned}
$$

in which $J_{3 / 2}(\xi a)$ is the Bessel function of the first kind of order $\frac{3}{2}$.

Now the displacement in $M_{2}$ is

$$
\begin{aligned}
V_{2}\left(x_{1}, x_{3}\right)= & \frac{1}{2 \pi} \int_{-\infty}^{\infty} \frac{4 \mu}{\bar{R}(\kappa)} e^{-p_{1} d}\left[1+\frac{4 h}{3 \pi} g(\kappa) e^{p_{1} d}\right] \\
& \times\left[M e^{p_{2} x_{3}-p_{3} H}+N e^{p_{3} x_{3}-p_{2} H}\right] e^{-i \eta x_{1}} d \xi \\
= & \frac{1}{2 \pi} \int_{-\infty}^{\infty} \frac{4 \mu e^{-p_{1} d}\left[M e^{p_{2} x_{3}-p_{3} H}+N e^{p_{3} x_{3}-p_{2} H}\right]}{\bar{R}(\kappa)\left[1-\frac{4 h}{3 \pi} g(\kappa) e^{p_{1} d}\right]} e^{-i \eta x_{1}} d \eta .
\end{aligned}
$$


Since the value of the above integral depends on the contribution of the poles of the integrand, the concerned dispersion equation is given by

$$
\bar{R}(\kappa)\left[1-\frac{4 h}{3 \pi} g(\kappa) e^{p_{1} d}\right]=0 .
$$

or,

$$
\begin{aligned}
& \left(\mu p_{1}+\mu^{\prime} p_{2}+\mu_{e} H_{3}^{2} p_{2}-i \eta \mu_{e} H_{1} H_{3}\right) M e^{-p_{3} H}+\left(\mu p_{1}+\mu^{\prime} p_{3}+\mu_{e} H_{3}^{2} p_{3}\right. \\
& \left.-i \eta \mu_{e} H_{1} H_{3}\right) N e^{-p_{2} H}-\frac{4 h}{3 \pi}\left[-p_{1} p_{2}\left(\mu-\mu^{\prime}-\mu_{e} H_{3}^{2}\right) M e^{-p_{3} H}\right. \\
& -p_{1} p_{3}\left(\mu-\mu^{\prime}-\mu_{e} H_{3}^{2}\right) N e^{-p_{2} H}-\left(\mu^{\prime} p_{2}^{2}-\mu p_{1}^{2}+\mu_{e} H_{3}^{2} p_{2}^{2}\right. \\
& \left.-i \eta \mu_{e} H_{1} H_{3} p_{2}+i \eta \mu_{e} H_{1} H_{3} p_{1}\right) M e^{-p_{3} H}-\left(\mu^{\prime} p_{3}^{2}-\mu p_{1}^{2}+\mu_{e} H_{3}^{2} p_{3}^{2}\right. \\
& \left.\left.-i \eta \mu_{e} H_{1} H_{3} p_{3}+i \eta \mu_{e} H_{1} H_{3} p_{1}\right) N e^{-p_{2} H}\right]=0 .
\end{aligned}
$$

After simplification one obtains the concerned dispersion equation as

$$
\tan \eta H \Gamma=\frac{N_{3}}{D_{3}}
$$

where

$$
\begin{aligned}
N_{3}= & \Gamma\left[R_{b}^{2}\left\{\frac{\mu c_{a}}{\mu^{\prime}}-\frac{4 h}{3 \pi} \eta\left(\frac{\mu c_{a}^{2}}{\mu^{\prime}}+\frac{R_{a}^{2} c_{b}^{2}}{R_{b}^{2}}\right)\right\}\right. \\
& \left.+\frac{\eta}{k_{0}}\left(\eta^{\prime 2}-\frac{c^{2}}{\beta_{2}^{2}}\right)\left\{R_{b}^{2}+\frac{4 h \eta c_{a}}{3 \pi}\left(\frac{\mu}{\mu^{\prime}}-R_{b}^{2}\right)\right\}\right]
\end{aligned}
$$

and

$$
\begin{aligned}
D_{3}= & \frac{R_{a}^{2} c_{b}^{2}}{R_{b}^{4}}\left[R_{b}^{4}+\frac{4 h \eta c_{a}}{3 \pi}\left\{\frac{\mu R_{b}^{2}}{\mu^{\prime}}-R_{b}^{4}\right\}\right] \\
& -\frac{\eta}{k_{0}}\left(\eta^{\prime 2}-\frac{c^{2}}{\beta_{2}^{2}}\right)\left\{\frac{\mu c_{a}}{\mu^{\prime}}-\frac{4 h \eta}{3 \pi}\left(\frac{\mu c_{a}^{2}}{\mu^{\prime}}+\frac{R_{a}^{2} c_{b}^{2}}{R_{b}^{2}}\right)\right\} .
\end{aligned}
$$

In the absence of irregularity of the interface $(h=0)$ the corresponding dispersion relation in presence of surface stress may be obtained from (45) as special case and is given by

$$
\tan \eta H \Gamma=\frac{N_{4}}{D_{4}} .
$$

In the absence of surface stress when the interface is irregular, the equation reduces

$$
\tan \eta H \Gamma=\frac{N_{5}}{D_{5}} .
$$


In the above the expressions for $N_{4}, D_{4}$ and $N_{5}, D_{5}$ are deduced from $N_{3}, D_{3}$ of (45) by simply putting $h=0$ and $\eta^{\prime 2}=c^{2} / \beta_{2}^{2}$ respectively.

It is observed from the equations (40) and (45) that the range of possible real values of $c$ for which the required wave may propagate i.e. the energy of plane waves be confined to the transition zone, is given by

$$
\beta_{2}<c<\beta_{1},
$$

where

$$
\beta_{2}=\sqrt{\frac{\mu^{\prime}}{\rho^{\prime}}}\left(1+\frac{\frac{\mu_{e} H_{1}^{2}}{\mu^{\prime}}}{1+\frac{\mu_{e} H_{3}^{2}}{\mu^{\prime}}}\right)^{\frac{1}{2}} .
$$

The in equation (48) indicates that the range depends on the magnetic field but does neither depend on the surface stress parameters nor on the interface irregularity. Hence, the presence of magnetic field alters the range. From the equations (42) and (47) it is interesting to note that the surface stress has no effect when $\eta^{\prime 2}=c^{2} / \beta_{2}^{2}$ i.e. when $c=\sqrt{\mu_{0} / \rho_{0}}$. Hence surface stress plays its role only when $c \neq \sqrt{\mu_{0} / \rho_{0}}$. In the absence of both the surface irregularity $(h=0)$ and the magnetic field $\left(H_{1}=0, H_{3}=0\right)$ the dispersion equation (40) and (45) reduces to the wave velocity equation for $\mathrm{SH}$-waves under the influence of surface stress as

$$
\tan \eta H\left(\frac{c^{2}}{\beta_{2}^{2}}-1\right)^{\frac{1}{2}}=\frac{\left(\frac{c^{2}}{\beta_{2}^{2}}-1\right)^{\frac{1}{2}}\left[\frac{\mu}{\mu^{\prime}}\left(1-\frac{c^{2}}{\beta_{2}^{2}} \frac{\beta_{2}^{2}}{\beta_{1}^{2}}\right)^{\frac{1}{2}}+\frac{\eta}{k_{0}}\left(\eta^{\prime 2}-\frac{c^{2}}{\beta_{2}^{2}}\right)\right]}{\left(\frac{c^{2}}{\beta_{2}^{2}}-1\right)-\frac{\eta}{k_{0}}\left(\eta^{\prime 2}-\frac{c^{2}}{\beta_{2}^{2}}\right) \frac{\mu}{\mu^{\prime}}\left(1-\frac{c^{2}}{\beta_{2}^{2}} \frac{\beta_{2}^{2}}{\beta_{1}^{2}}\right)^{\frac{1}{2}}},
$$

where $\beta_{2}^{2}=\frac{\mu^{\prime}}{\rho^{\prime}}, k_{0}=\frac{\mu^{\prime}}{\rho_{0} \beta_{2}^{2}}, \eta^{\prime 2}=\frac{\mu_{0}}{\rho_{0} \beta_{2}^{2}}$, which is in conformity with the corresponding result obtained by Pal et al (1997).

Again if one considers the effect of irregularity only, neglecting the effect of surface stress and magnetic field the equation (45) transforms to the result obtained by Chattopadhyay \& Pal (1983).

\section{Numerical calculations}

For the purpose of numerical calculation and graphical representation we take (Pal et al 1997)

$$
\begin{aligned}
& \mu=3.00 \times 10^{6} \mathrm{~N} / \mathrm{cm}^{2}, \mu^{\prime}=5.00 \times 10^{6} \mathrm{~N} / \mathrm{cm}^{2}, \mu_{0}=6.47 \times 10^{6} \mathrm{~N} / \mathrm{cm} . \\
& \rho=2.72 \mathrm{gm} / \mathrm{cm}^{3}, \rho^{\prime}=9.89 \mathrm{gm} / \mathrm{cm}^{3}, \rho_{0}=3.40 \mathrm{gm} / \mathrm{cm}^{2} .
\end{aligned}
$$

To highlight the effect of magnetic field, irregular boundary and surface stress in the case of plane SH waves propagating in a homogeneous, perfectly conducting crustal layer lying over an isotropic solid half space we draw the curves (figures 3 to 10). With the help of these figures comparison may also be made among different cases. Using the frequency equations (40) and (45) numerical values of $c / \beta_{2}$ have been plotted against $\eta H$ for different cases as expressed in the captions of the figures. All the figures are self explanatory and contain some important peculiarities due to the effect of magnetic field, non-parallel boundary, surface 


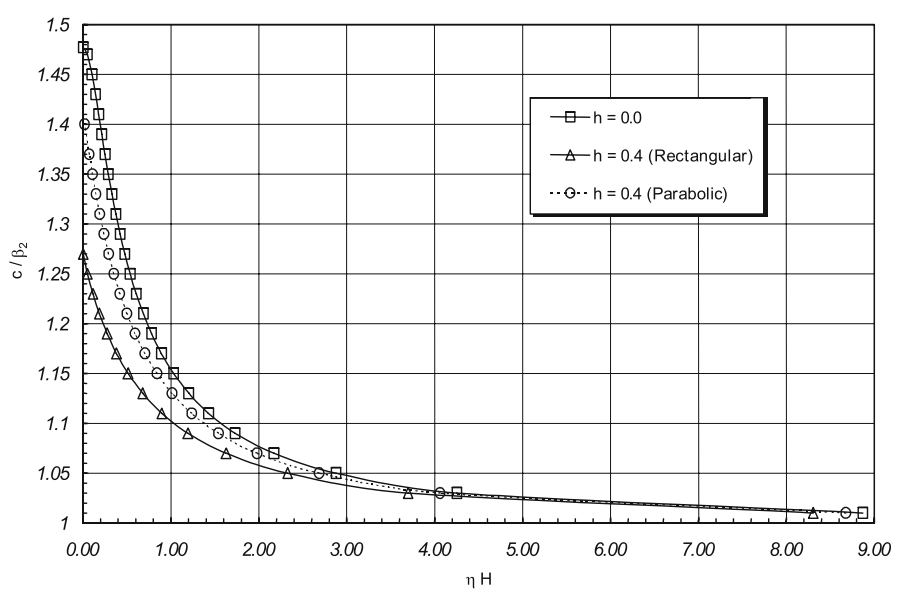

Figure 3. $c / \beta_{2}$ vs $\eta H$ for $H_{1}=0, H_{3}=0$ and surface stress $=0 \cdot 0$.

stress on the surface $x_{3}=-H$. However, for better understanding of the figures the following explanations are presented.

Figure 3 expresses the variations of $c / \beta_{2}$ for different values of $\eta H$ in the absence of magnetic field and surface stress. Two curves of this figure have been drawn to highlight the variation of wave velocity due to rectangular and parabolic irregularities with $h=0.4$ in each case. The third one which corresponds to no irregularity $(h=0)$, has been drawn for comparison. It is seen that, in each case, the values of $c / \beta_{2}$ start from its highest value when $\eta H=0$ and it decreases sharply as $\eta H$ increases to a certain value. After this particular value of $\eta H$ the decrement in $c / \beta_{2}$ due to the increase of $\eta H$ is very small. It is marked from this figure that irregularity of any nature always reduces wave velocity (with respect to the no irregularity case) to a certain extent. For a particular value of $\eta H$ the rectangular irregularity causes more reduction of wave velocity than that of parabolic irregularity.

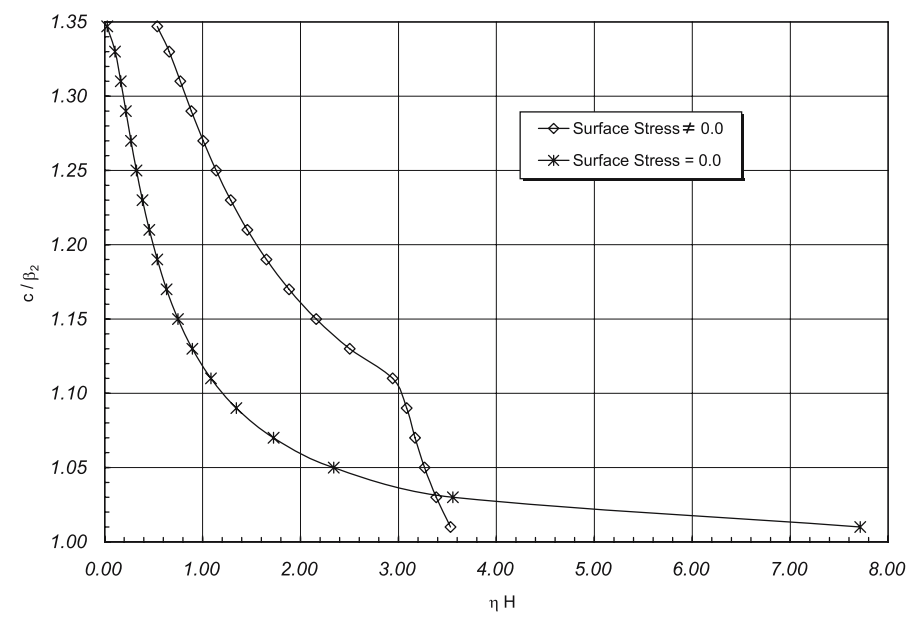

Figure 4. $c / \beta_{2}$ vs $\eta H$ for $h=0 \cdot 0$ and $\mu_{e} H_{1}^{2} / \mu^{\prime}=0 \cdot 2, H_{3}=0$. 


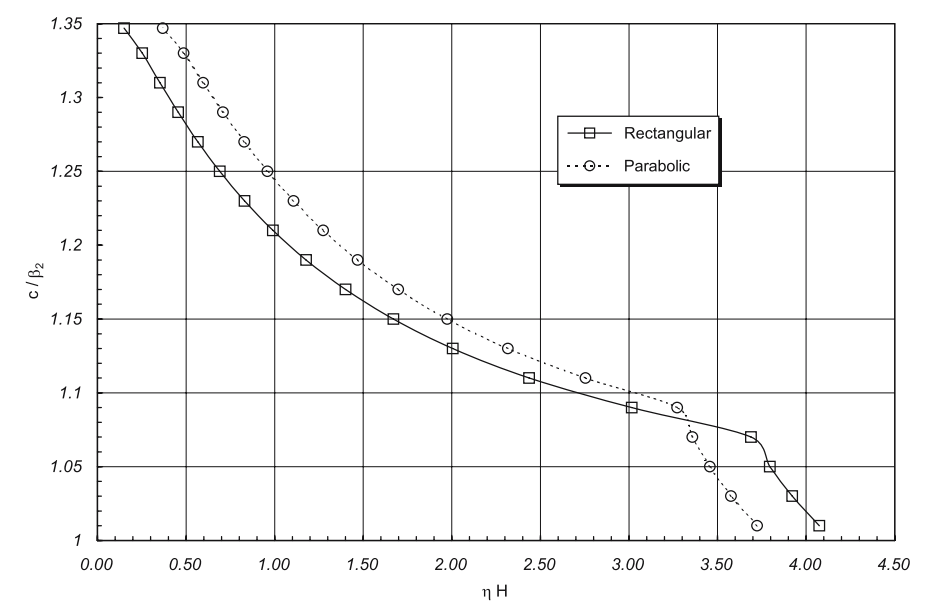

Figure 5. $c / \beta_{2}$ vs $\eta H$ for $h=0 \cdot 4$, surface stress $\neq 0 \cdot 0$ and $\mu_{e} H_{1}^{2} / \mu^{\prime}=0 \cdot 2, H_{3}=0$.

In figure 4 we have considered the case of no irregularity with horizontal magnetic field $\left(\mu_{e} H_{1}^{2} / \mu^{\prime}=0 \cdot 2\right)$ in presence of surface stress. For the sake of comparison, a curve has been plotted corresponding to the boundary which is free of surface stress. We see that there is a sudden fall of wave velocity after $\eta H=3$. It is worthy to note that surface stress causes increment of wave velocity up to $\eta H<3.4$. When $\eta H=3.4$ surface stress has no effect on the propagation of $\mathrm{SH}$-waves under the influence of magnetic field. When $\eta H>3.4$ surface stress causes sharp diminution of wave velocity.

Figure 5 gives the variation of $c / \beta_{2}$ with respect to $\eta H$ under the combined effect of horizontal magnetic field and surface stress with rectangular or parabolic irregularity. Here also it is noted that for a particular value of $\eta H$ wave velocity in the case of parabolic irregularity is greater than that of rectangular irregularity up to $\eta H=3 \cdot 3$. When $\eta H=3 \cdot 3$ there is no variation of wave velocity due to rectangular and parabolic irregularity. When

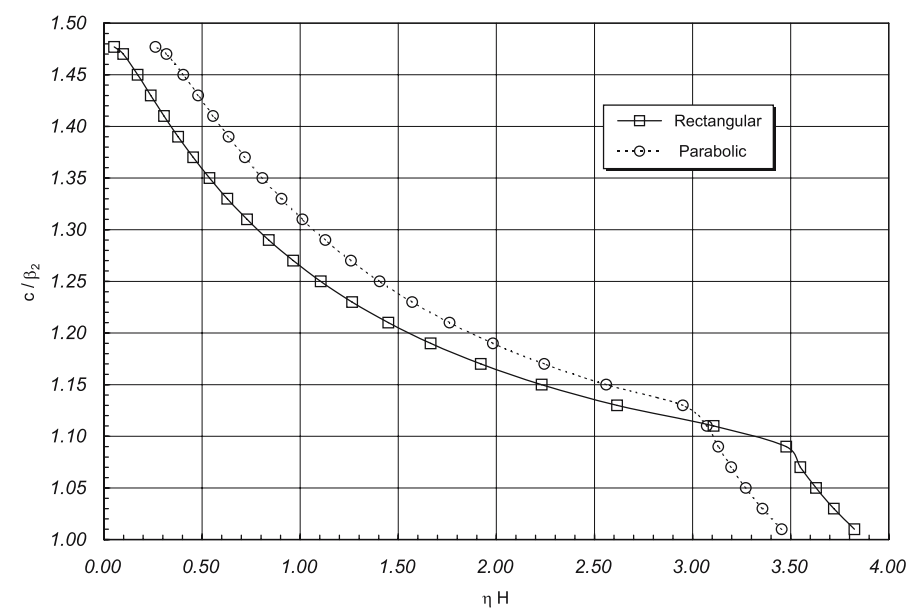

Figure 6. $c / \beta_{2}$ vs $\eta H$ for $h=0.4$, surface stress $\neq 0 \cdot 0$ and $H_{1}=0, H_{3}=0$. 


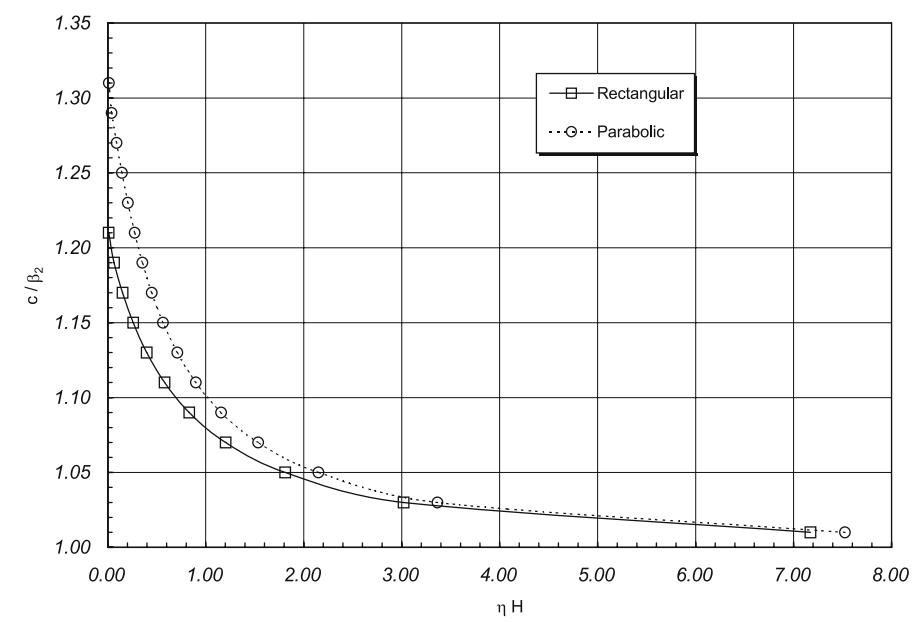

Figure 7. $c / \beta_{2}$ vs $\eta H$ for $h=0.4$, surface stress $=0.0$ and $\mu_{e} H_{1}^{2} / \mu^{\prime}=0 \cdot 2, H_{3}=0$.

$\eta H>3 \cdot 3$, parabolic irregularity causes more diminution of wave velocity than that of rectangular irregularity. Sudden fall occurs just after $\eta H=3.3$ and $\eta H=3.7$ for parabolic and rectangular irregularity respectively.

From figure 6 combined effect of surface stress and irregularity of interface can be studied when there is no magnetic field. Figure 7 depicts the combined effect of horizontal magnetic field and irregular interface in the case of rectangular and parabolic irregularity.

Figure 8 shows the variation of $c / \beta_{2}$ with respect to $\eta H$ under the joint influence of vertical magnetic field and surface stress when there is no irregularity in the interface. The curve corresponding to surface stress equal to zero, in this case, has also been plotted. Figure 9 gives us the modulation of $c / \beta_{2}$ under the combined effect of surface stress, irregularity of the interface and vertical magnetic field. Figure 10 gives us the modulation of wave velocity with respect to $\eta H$ under the joint effect of irregularity and vertical magnetic field. Cases

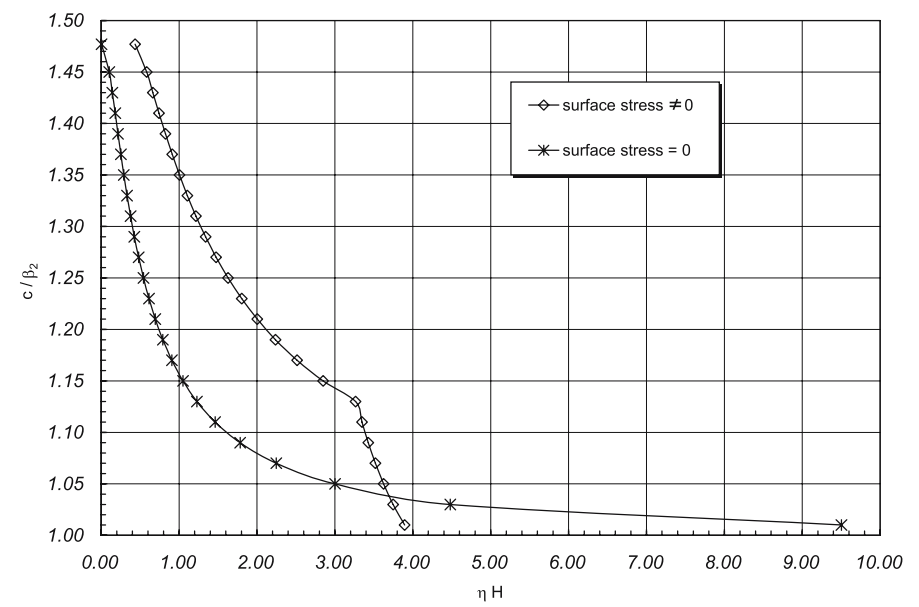

Figure 8. $c / \beta_{2}$ vs $\eta H$ for $h=0 \cdot 0$ and $\mu_{e} H_{3}^{2} / \mu^{\prime}=0 \cdot 2, H_{1}=0$. 


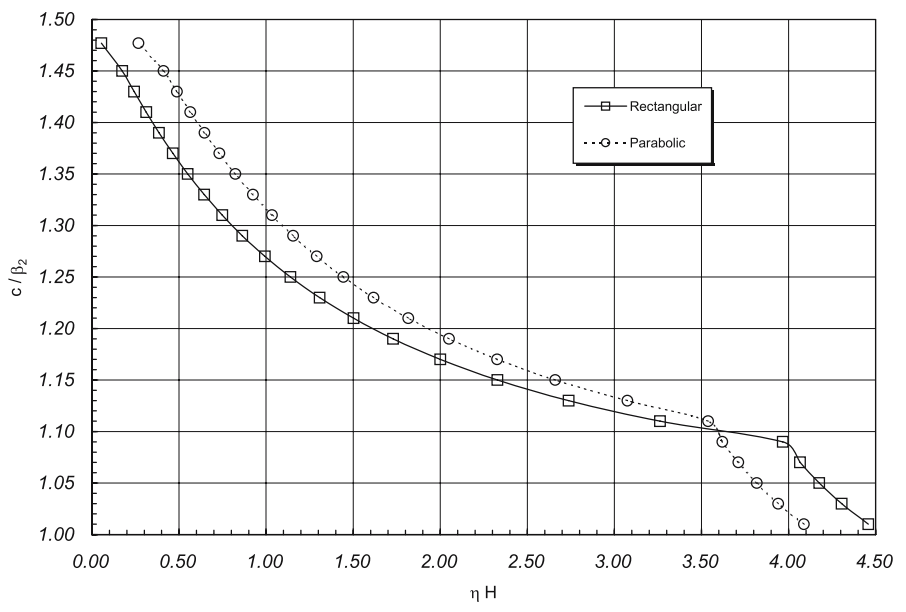

Figure 9. $c / \beta_{2}$ vs $\eta H$ for $h=0 \cdot 4$, surface stress $\neq 0 \cdot 0$ and $\mu_{e} H_{3}^{2} / \mu^{\prime}=0 \cdot 2, H_{1}=0$.

of parabolic and rectangular irregularity have been plotted separately. A close observation of all the figures leads us to express that the sudden fall of wave velocity occurs only when the surface stress is present on the surface $x_{3}=-H$.

\section{Conclusion}

The most significant outcome of this paper is that modulations of SH wave velocity takes place due to the presence of surface stress, magnetic field and irregularity near the interface. Further modulation take place due to their combined effect. In conclusion we also point out the peculiarity that a sudden fall of the wave velocity occurs after a certain value of $\eta H$ in all the cases where surface stress is present. Moreover, there exists a value (we call it critical

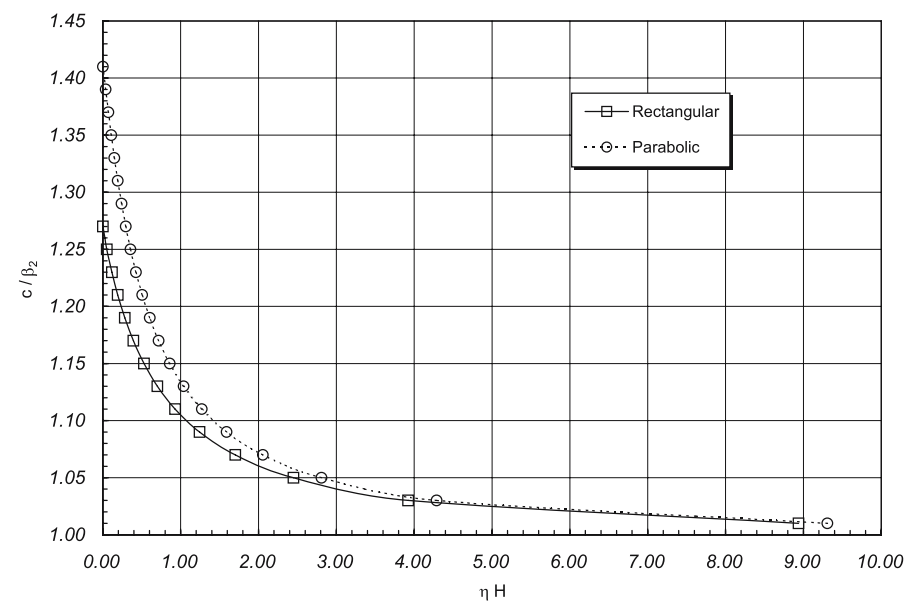

Figure 10. $c / \beta_{2}$ vs $\eta H$ for $h=0 \cdot 4$, surface stress $=0 \cdot 0$ and $\mu_{e} H_{3}^{2} / \mu^{\prime}=0 \cdot 2, H_{1}=0$. 
value) of $\eta H$ for which surface stress has no role to play. When $\eta H$ is less than this critical value, surface stress causes increment of wave velocity. On the other hand, when $\eta H$ is greater than this critical value surface stress causes decrement in wave velocity. The present problem and the results obtained have their possible applications in the field of earth sciences, geophysics, seismology specially to the problems of waves and vibrations where the wave signals have to travel through different layers exhibiting surface stresses due to material properties and containing irregularities due to continental margin, mountain roots, etc.

Thanks are due to Sri Sourav Acharya, Scientific Officer, Atomic Energy Regulatory Board, Mumbai, for his computational help towards the numerical calculations and graphs. Thanks are also due to the reviewers of this paper for their valuable suggestions.

\section{References}

Bazer J 1984 A survey of linear and nonlinear waves in a perfect magneto-elastic medium in the mechanical behaviour of electromagnetic solid continua, (ed.) G A Maugin, (B.V. (North-Holland): Elsevier Science Publishers)

Bhattacharya J 1962 On the dispersion curve of Love waves due to irregularity in the thickness of the transversely isotropic crustal layer. Gerl. Beitr. Geophys. Leipzig 71: 324-333

Chandrasekharaih D S 1987a Effect of surface stresses and voids on Rayleigh waves in an elastic solid. Int. J. Eng. Sci. Great Britain 25: 205-211

Chattopadhyay A 1975a On the dispersion equation for Love waves due to irregularity in the thickness of the non-homogeneous crustal layer. Acta Geophysica Polonica. 23: 307-317

Chattopadhyay A, Choudhury S, Chattopadhyay A 1998 Diffraction of magneto-elastic shear waves in a self-reinforced medium by a rigid strip. Ind. J. Pure Appl. Math. 29: 81-94

Chattopadhyay A, Chowdhury S 1995 Magneto-elastic shear waves in an infinite self-reinforced plate. Int. J. Numer. Anal. Methods Geomech. 19: 289-304

Chattopadhyay A, Pal A K 1983 Dispersion curves of SH waves caused by irregularity in the prestressed internal stratum. Acta Geophysica Polonica. XXXI: 37-49

Chaudhary S, Kaushik V P, Tomar S K 2004 Reflection/transmission of plane SH wave through a self-reinforced elastic layer between two half-spaces. Acta Geophysica Polonica. 52: 219-235

Coulomb J 1952 Love waves of the Queen Charlotte Islands earthquake of August 22, 1949. Bull. Seism. Soc. Amer. 42: 29-36

De Noyer J 1961 The effect of variations in layer thickness of Love waves. Bull. Seism. Soc. Amer. 51: 227-235

Dunkin J W, Eringen A C 1963 On the propagation of waves on electromagnetic elastic solids. Int. J. Engng. Sci. 1: 461-495

Dutta S 1963a Love waves in a non-homogeneous internal stratum lying between two semi-infinite isotropic media. Geophysics. 28: 156-160

Dutta S 1963b On the propagation of Love waves in a non-homogeneous internal stratum of finite depth lying between two semi-infinite isotropic media. Geophys. Pure Appl. 55: 31-36

Gurtin M E 1972 The linear theory of elasticity. Handbuch der Physik. Springer, Berlin. VIa/3

Gurtin M E, Murdoch A I 1974-75 A continuum theory of elastic material surfaces. Arch. Rat. Mech. Anal. 57: 291-232

Gurtin M E, Murdoch A I 1976 Effect of surface stress on wave propagation in solids. J. Appl. Phys. 47: 4414-4429

Kar B K 1977 On the propagation of Love type waves in a non-homogeneous internal stratum of finite thickness lying between two semi-infinite isotropic elastic media. Geophysik. Leipzig. 86: 407-412 
Knopoff L 1955 The interaction between elastic wave motion and a magnetic field in electrical conductors. J. Geophys. Res. 60: 441-456

Lee J S, Its E N 1992 Propagation of Rayleigh waves in magneto-elastic media, Trans. ASME, J. Appl. Mech. 59: 812-818

Mal A K 1962 On the frequency equation for Love waves due to abrupt thickening of the crustal layer. Geophys. Pure Appl. 52: 59-68

Maugin G A 1988b 'Shear horizontal surface acoustic waves on solids' in recent advances in surface acoustic waves. (eds) D F Parker, G A Maugin (Berlin: Springer-Verlag) 158-172

Othman M I A, Song Y 2006 The effect of rotation on the reflection of magneto-thermo-elastic waves under thermo-elasticity without energy dissipation. Acta Mechanica. 184: 189-204

Pal P K, Acharya D P, Sengupta P R 1997 Effect of surface stresses on surface waves in elastic solids. Sādhanā 22: 659-670

Plaster H J 1972 Blast cleaning and Allied Processes. Industrial NewsPapers Ltd. London

Sato Y 1952 Study of surface waves V, Love waves propagated upon heterogeneous medium. Bull. Earthquake Res. Inst. Tokyo. 30: 1

Stoneley R 1924 Elastic waves at the surface of separation of two solids. Proc. Roy. Soc. London, A106: 416-428

Tomita S, Shindo Y 1979 Rayleigh waves in magneto-thermo-elastic solid with thermal relaxation. Int. J. Eng. Sci. 17: 227-232

Tranter C J 1966 Integral transforms in mathematical physics: 63-67

Verma P D S 1986 Magneto-elastic shear waves in self-reinforced bodies. Int. J. Eng. Sci. 24: 1067-1073

Verma P D S, Rana O H, Verma M 1988 Magneto-elastic transverse surface waves in self-reinforced elastic solids. Indian J. Pure Appl. Math. 19: 713-716

Willis H F 1948 A formula for expanding an integral as a series, Philosophical Mag. 39: 455-459

Wolf B 1967 Propagation of Love waves in surface layers of varying thickness. Pure Appl. Geophys. 67: 76-82

Yu Chia P, Tang Sam 1966 Magneto-elastic waves in initially stressed conductors. J. Appl. Math. Phys. ZAMP. 17: 766-775 